\title{
Factores asociados a mortalidad en pacientes con falla cardiaca descompensada*
}

\author{
Factors associated with mortality in patients with \\ decompensated heart failure
}

\author{
Walter Gabriel Chaves, Juan José Diaztagle, John Jaime Sprockel, \\ José IgnaCio HERnández, JaVIER MAURICIO BenAVIDEZ, \\ Diana Cristina Henao, María Gimena Mejía, Viviana Vargas, \\ Nubia Esperanza Carrero, Carmelo Rafael Fuentes, \\ Magda Janeth Alba, Ricardo Andrés Carvajal, Fabio Andrés Contento \\ - Bogotá, D.C. (Colombia)
}

\section{Resumen}

Introducción: la falla cardiaca (FC) es una de las principales causas de morbimortalidad a nivel mundial, la cual ha experimentado aumento gradual de su incidencia sin variación importante en su desenlace en las dos últimas décadas. En Colombia muy pocos estudios evalúan factores asociados a mortalidad por falla cardiaca.

Métodos: estudio de cohorte prospectivo en el que se incluyeron pacientes con diagnóstico de falla cardiaca descompensada al momento del ingreso a urgencias, entre febrero de 2010 y marzo de 2013. Se calculó el tamaño de muestra y se realizó un análisis multivariado para la evaluación de los factores de riesgo asociados a mortalidad intrahospitalaria y a 30 días.

Resultados: se incluyeron 462 pacientes. La mortalidad hospitalaria fue de $8.9 \%$ y a 30 días de $13.8 \%$, en el modelo multivariado para el desenlace mortalidad intrahospitalaria se observó que la única variable con significancia estadística fue el BUN $\geq 43 \mathrm{mg} / \mathrm{dL}$ (OR, 3.45 [IC 95\% 1.54-7.74], $\mathrm{p}=0.003$ ). Para la mortalidad a 30 días, la estancia hospitalaria $>5$ días (OR, 2.23 [IC 95\% 1.204.12], $\mathrm{p}=0.011)$, el BUN $\geq 43 \mathrm{mg} / \mathrm{dL}(\mathrm{OR}, 2.55$ [IC 95\% 1.31-4.94], $\mathrm{p}=0.005$ ) y el NT-proBNP $\geq$ $4630 \mathrm{pg} / \mathrm{dL}$ (OR, 2.47 [IC 95\% 1.30-4.70], p=0.006).

Conclusiones: la mortalidad intrahospitalaria de los pacientes con falla cardiaca descompensada en la población evaluada fue alta. En los análisis multivariados, se encontró que el BUN $\geq 43 \mathrm{mg} / \mathrm{dL}$ fue el único factor de riesgo independiente asociado a mortalidad intrahospitalaria; mientras que la mortalidad a 30 días se relacionó además con el NT-proBNP y la estancia hospitalaria superior a cinco días. (Acta Med Colomb 2014; 39: 314-320)

Palabras clave: falla cardiaca, mortalidad, factores de riesgo, NT-ProBNP

\section{Abstract}

Introduction: heart failure is one of the main causes of morbidity and mortality worldwide; it has experienced a gradual increase in incidence with no significant variation in outcome in the last two decades. In Colombia there are no studies to evaluate risk factors for mortality, which is the subject of this study.

Methods: prospective cohort study in which patients with diagnosis of decompensated heart failure on admission to the emergency department between February 2010 and March 2013 were included. The sample size was calculated and a multivariate analysis was performed to evaluate the risk factors associated with in-hospital and 30-day mortality.

Results: 462 patients were included. Hospital mortality was $8.9 \%$ and 30-day mortality $13.8 \%$; in the multivariate model for hospital mortality outcome was observed that the only variable with statistic significance was BUN $\geq 43 \mathrm{mg} / \mathrm{dL}$ (OR, 3.45 [95\% CI 1.54- 7.74], $\mathrm{p}=0.003$ ). For 30day mortality, hospital stay $>5$ days (OR, 2.23 [95\% CI 1.20-4.12], p = 0.011), BUN $\geq 43 \mathrm{mg} /$ $\mathrm{dL}(\mathrm{OR}, 2.55$ [95\% CI 1.31-4.94] , p = 0.005) and NT-proBNP $\geq 4630 \mathrm{pg} / \mathrm{dL}(\mathrm{OR}, 2.47$ [95\% CI $1.30-4.70], \mathrm{p}=0.006)$.
* Trabajo ganador del premio al "Mejor Trabajo de Investigación Clínica", otorgado por el XXIII Congreso Colombiano de Medicina Interna. Cartagena de Indias, 7-10 de agosto de 2014. Dr. Walter Gabriel Chaves Santiago: Internista. Profesor Asistente y Jefe de Posgrado de Medicina Interna, Fundación Universitaria de Ciencias de la Salud. Jefe Servicio de Medicina Interna Hospital de San José. Especialista en Docencia e Investigación. Magister en Docencia e Investigación; Dr. Juan José Diaztagle Fernández: Internista, Epidemiólogo, Magister en Fisiología. Instructor de Medicina Interna, Fundación Universitaria Ciencias de la Salud Hospital de San José. Profesor Asociado Departamento de Ciencias Fisiológicas, Universidad Nacional de Colombia; Dr. John Jaime Sprockel Díaz: Internista, Candidato a Maestría en Ingeniería de Sistemas y Computación Pontificia Universidad Javeriana. Instructor de Medicina Interna Fundación Universitaria de Ciencias de la Salud - Hospital de San José; Dr. José Ignacio Hernández Cruz: Internista, Cardiólogo. Profesor Emérito de Medicina Interna, Fundación Universitaria de Ciencias de la Salud - Hospital de San José; Dr. Javier Mauricio Benavides Bermúdez: Internista, Instructor de Medicina Interna, Fundación Universitaria de Ciencias de la Salud - Hospital de San José; Dra. Diana Cristina Henao Carrillo: Internista, Endocrinóloga, Fundación Universitaria de Ciencias de la Salud - Hospital de San José; Dres. María Gimena Mejía López, Viviana Vargas Buitrago, Nubia Esperanza Carrero Rojas y Carmelo Rafael Fuentes Restrepo: Internistas Fundación Universitaria de Ciencias de la Salud - Hospital de San José; Dra. Magda Janeth Alba Saavedra: División de Investigaciones, Fundación Universitaria de Ciencias de la Salud - Hospital de San José; Dres. Ricardo Andrés Carvajal Flechas y Fabio Andrés Contento Anaya: Residentes Segundo Año de Medicina Interna, Fundación Universitaria de Ciencias de la Salud - Hospital de San José. Bogotá D.C., (Colombia).

Correspondencia. Dr. Walter Gabriel Chaves. Bogotá D.C., (Colombia).

E-mail:wgchs1973@gmail.com

Recibido: 19/IX/2014 Aceptado: 6/XI/2014 
Conclusions: in-hospital mortality in patients with decompensated heart failure in the study population was high. In multivariate analysis, it was found that $\mathrm{BUN} \geq 43 \mathrm{mg} / \mathrm{dL}$ was the only independent risk factor associated with hospital mortality, while the 30-day mortality was also associated with NT-proBNP and hospital stay greater than five days. (Acta Med Colomb 2014; 39: 314-320)

Keywords: heart failure, mortality, risk factors, NT-proBNP

\section{Introducción}

La falla cardiaca (FC) representa una de las mayores causas de morbilidad y mortalidad a nivel mundial (1). En países desarrollados, esta entidad afecta entre 1 y $2 \%$ del total de la población adulta, con una prevalencia que alcanza $10 \%$ en mayores de 70 años (2). En Estados Unidos, 5.1 millones de personas tienen falla cardiaca clínicamente manifiesta y se ha estimado que las personas mayores de 20 años tienen una probabilidad de $20 \%$ de desarrollar esta enfermedad a lo largo de la vida (3). En Latinoamérica el incremento en la expectativa de vida, la alta prevalencia de enfermedad coronaria, diabetes mellitus e hipertensión arterial (HTA), asociado a patologías como la enfermedad de Chagas y la cardiopatía reumática, han dado como resultado un aumento en la incidencia de esta entidad en la población adulta mayor de 65 años $(4,5)$. Esta región del mundo está experimentando una "epidemia" a gran escala de factores de riesgo para FC (6), lo cual se agrava por un menor gasto per capita en salud que limita el acceso a estrategias preventivas y terapéuticas adecuadas (7).

En el curso de esta patología, la calidad de vida y el pronóstico a largo plazo son desalentadores, con una supervivencia a cinco años que se asemeja con las enfermedades de origen neoplásico (8). Registros internacionales han mostrado que la mortalidad intrahospitalaria se encuentra entre 4 y $7 \%$, con medianas de estancias hospitalarias entre 4 y 9 días (9-11). En Latinoamérica, dos registros han mostrado mortalidad de $5.6 \%$ y $8 \%$, mientras que la estancia hospitalaria fue de $10 \pm$ 9 días en promedio, con una mediana de siete días $(12,13)$.

Se han documentado varios factores de riesgo relacionados con la mortalidad entre los cuales se encuentran: edad, frecuencia cardiaca, presión sanguínea, sodio sérico, nitrógeno ureico, raza y enfermedad pulmonar obstructiva crónica (EPOC). El reconocimiento de estos factores durante la hospitalización es fundamental, por lo que se recomienda utilizar puntajes para estratificar el riesgo de mortalidad en pacientes hospitalizados $(2,3)$.

En Colombia no se cuenta con mucha información acerca de los aspectos epidemiológicos de la FC (14-17). Por lo tanto, el objetivo del presente estudio fue describir una población de pacientes hospitalizados con FC descompensada y determinar los factores de riesgo asociados a mortalidad hospitalaria y a 30 días.

\section{Metodología}

Se realizó un estudio de cohorte prospectivo. Los pacientes elegibles fueron aquellos con diagnóstico de FC descompensada hospitalizados por el servicio de medicina interna, en un hospital universitario de cuarto nivel durante el periodo comprendido entre febrero 2010 y marzo 2013. Se incluyeron pacientes mayores de 18 años que tuvieran como diagnóstico principal FC descompensada y cumplieran con los criterios de Framingham (dos criterios mayores o un criterio mayor y dos criterios menores)(18). Se excluyeron pacientes con cetoacidosis diabética o estado hiperosmolar, urgencia dialítica, cirrosis Child $\mathrm{C}$, insuficiencia hepática aguda, síndrome nefrótico, choque hipovolémico o séptico y neoplasia terminal.

Se diseñó un formato para verificar criterios de elegibilidad y se recolectó la información en un formato estructurado en donde se anotaron datos de variables sociodemográficas, clínicas, comorbilidades, hospitalizaciones previas por FC y datos del examen físico. Se tomaron muestras para determinar niveles de BUN, creatinina, NT-proBNP (sistema de inmunodiagnóstico 5600 Integrated System), hemoglobina, sodio y troponina I (técnica inmunométrica leídas por sistema Vitros) y un electrocardiograma al ingreso. La fracción de eyección del ventrículo izquierdo (FEVI) se obtuvo a partir del ecocardiograma realizado por el servicio de cardiología institucional o estudios previos no mayores a tres meses.

Los pacientes se siguieron hasta el egreso hospitalario. Se documentó la mortalidad intrahospitalaria y mediante llamada telefónica o verificación del RUAF (registro único de afiliados), la mortalidad a 30 días.

\section{Análisis estadístico}

Se utilizó el programa STATA 12, las variables continuas se expresan con medidas de tendencia central (promedios o medianas) y dispersión (desviación estándar y rangos intercuartílicos), las variables categóricas con frecuencias absolutas y relativas. Se realizó un cálculo del tamaño para el desenlace mortalidad intrahospitalaria con una proporción de eventos esperados de $5 \%$, capacidad de detección de OR de 1.7 nivel de significancia de $0.05 \mathrm{y}$ un poder de 0.8 (19). El tamaño calculado inicialmente fue de 481 pacientes. Un análisis realizado en marzo de 2013 evidenció una mortalidad mayor que la esperada, por lo cual se consideró que se tenía un tamaño de muestra suficiente $(n=462)$ para el objetivo de la investigación bajo la regla empírica de Freeman, la que se exige que haya al menos 10 eventos por cada variable incluida en el modelo (20).

Se categorizaron las siguientes variables: frecuencia cardiaca $(\geq 70 \mathrm{lpm})$, presión arterial sistólica $(<115 \mathrm{mmHg})$, creatinina $(>2 \mathrm{mg} / \mathrm{dL})$, anemia $(\mathrm{Hb}<12 \mathrm{~g} / \mathrm{dL})$ y BUN $\geq 43$ 
$\mathrm{mg} / \mathrm{dL}$. Se definió hiponatremia $(\mathrm{Na}<135 \mathrm{mEq} / \mathrm{L})$, FEVI reducida $(<40 \%)$ y estancia prolongada $>5$ días.

Se realizó un análisis de regresión logística multivariado por pasos. Primero se hizo análisis bivariado que incluyó las siguientes variables: edad, sexo, frecuencia cardiaca $\geq 70$ $1 \mathrm{pm}$, presión arterial sistólica $<115 \mathrm{mmHg}$, hiponatremia, creatinina $>2 \mathrm{mg} / \mathrm{dL}, \mathrm{BUN} \geq 43 \mathrm{mg} / \mathrm{dL}, \mathrm{Hb}<12 \mathrm{~g} / \mathrm{dL}$, troponina I positiva $(>0.4 \mathrm{pcg} / \mathrm{mL})$, FEVI reducida, estancia hospitalaria prolongada, niveles de TN-pro-BNP (se utilizó la mediana) y antecedentes de enfermedad coronaria, EPOC, diabetes mellitus tipo 2, HTA, fibrilación auricular, hospitalización previa por FC, uso previo de betabloqueadores (BB). Seguidamente se realizó un análisis multivariado con inclusión "hacia adelante"con las variables que en el análisis bivariado presentaron significancia estadística utilizando la prueba de chi cuadrado definidas por un valor $\mathrm{p}<0.05$, y con variables que se consideraron clínicamente significativas.

Este estudio fue aprobado por el comité de ética e investigación en humanos de la Facultad de Medicina y el hospital donde se realizó la investigación. Se consideró que no fue necesario de consentimiento informado. Recibió financiación por parte de la convocatoria interna N 4-2009.

\section{Resultados}

Entre febrero de 2010 y marzo de 2013 ingresaron 485 pacientes, de los cuales se excluyeron 23 (Figura 1), por lo tanto la muestra total fue de 462. Las características demográficas se exponen en la Tabla 1. El promedio de edad fue de 72.4 (DE 12.7) años, 240 (51.9\%) fueron mujeres, $372(80 \%)$ tuvieron HTA, $202(43.7 \%)$ EPOC, $108(23.3 \%)$ diabetes mellitus, 87 (18.8\%) enfermedad coronaria y 223 (47.3\%) estuvieron hospitalizados previamente por falla cardiaca descompensada.

En cuanto al tratamiento recibido por estos pacientes, 190 $(41.1 \%)$ recibían betabloqueador, 155 (23.1\%) inhibidores de

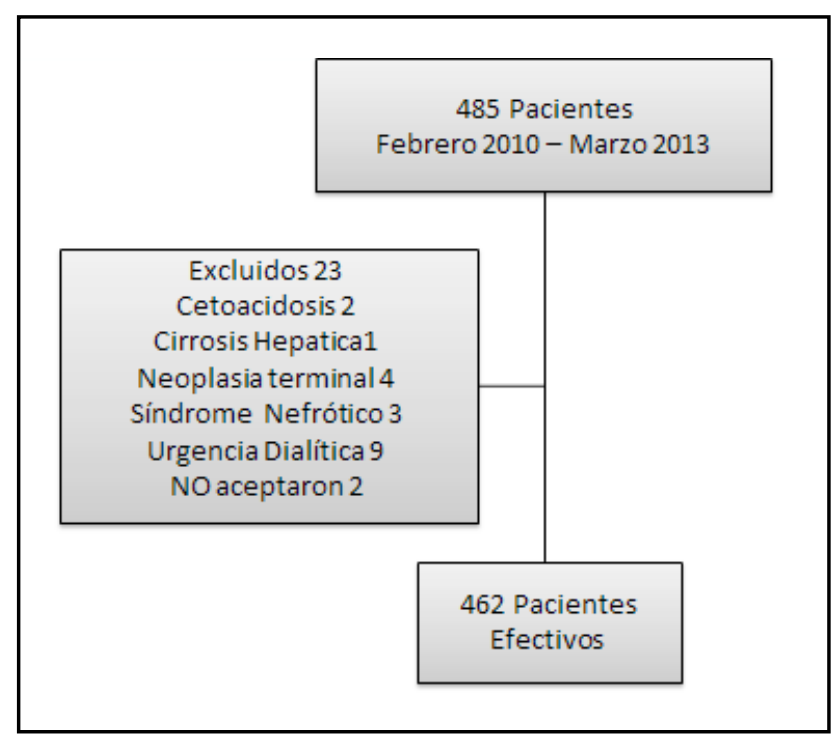

Figura 1. Diagrama de flujo de los pacientes seleccionados para el estudio.
Tabla 1. Características demográficas de los participantes del estudio, $n(=462)$.

\begin{tabular}{|c|c|c|}
\hline $\begin{array}{l}\text { Edad, años, promedio (DE) } \\
\text { Sexo femenino, n (\%) }\end{array}$ & $\begin{array}{r}72.4 \\
240\end{array}$ & $\begin{array}{l}(12.7) \\
(51.9)\end{array}$ \\
\hline \multicolumn{3}{|l|}{ Comorbilidades, n (\%) } \\
\hline HTA & 372 & $(80.5)$ \\
\hline Enfermedad coronaria & 87 & $(18.8)$ \\
\hline Diabetes mellitus tipo 2 & 108 & $(23.3)$ \\
\hline Enfermedad renal crónica* & 66 & $(14.2)$ \\
\hline EPOC & 202 & $(43.7)$ \\
\hline Fibrilación auricular & 84 & $(18.1)$ \\
\hline \multicolumn{3}{|l|}{ Hallazgos al examen físico } \\
\hline TAS, promedio (DE) $\mathrm{mmHg}$ & 131.6 & (26.4) \\
\hline $\mathrm{TAD}$, promedio (DE) $\mathrm{mmHg}$ & 77.1 & (15.4) \\
\hline Frecuencia cardiaca, promedio (DE) lpm & 85.8 & $(21.2)$ \\
\hline Frecuencia respiratoria, promedio (DE) rpm & 20.9 & $(4.8)$ \\
\hline \multicolumn{3}{|l|}{ Hallazgos paraclínicos } \\
\hline Creatinina, mediana (RIQ) $\mathrm{mg} / \mathrm{dL}$ & 1.0 & $(0.8-1.4)$ \\
\hline Creatinina $>2 \mathrm{mg} / \mathrm{dL}, \mathrm{n}(\%)$ & 47 & $(10.2)$ \\
\hline Sodio, promedio $(\mathrm{DE}) \mathrm{mEq} / \mathrm{L}$ & 138.1 & $(5.5)$ \\
\hline Hiponatremia $\dagger, \mathrm{n}(\%)$ & 98 & $(21.2)$ \\
\hline BUN, mediana (RIQ) mg/dL & 24 & $(18-35)$ \\
\hline $\mathrm{BUN} \geq 43 \mathrm{mg} / \mathrm{dL}$ & 74 & $(16.3)$ \\
\hline Troponina I, mediana(RIQ) $\mu \mathrm{g} / \mathrm{dL}$ & 0.04 & $(0.015-0.09)$ \\
\hline Troponinas positivas $\$, \mathrm{n}(\%)$ & 41 & $(9.7)$ \\
\hline NT-proBNP§, mediana (RIQ) pg/mL & 4630 & $(1780-12068)$ \\
\hline NT-proBNP $>125 \mathrm{pg} / \mathrm{mL}, \mathrm{n}(\%)$ & $426 / 438$ & $(97.2)$ \\
\hline Hemoglobina, promedio (DE) g/dL & 13,6 & $(2.9)$ \\
\hline Hb menor $12 \mathrm{~g} / \mathrm{dL}, \mathrm{n}(\%)$ & 144 & $(31.1)$ \\
\hline \multicolumn{3}{|l|}{ Hallazgos ecocardiográficos } \\
\hline FEVI, menor del $40 \%$ J, n (\%) & 143 & $(32.9)$ \\
\hline \multicolumn{3}{|l|}{ Valvulopatía **, n (\%) } \\
\hline Aórtica & 140 & $(32.2)$ \\
\hline Tricuspídea & 140 & $(32.2)$ \\
\hline Mitral & 245 & $(56.4)$ \\
\hline \multicolumn{3}{|l|}{ Clase funcional base, n (\%) } \\
\hline I & 79 & $(17.1)$ \\
\hline II & 253 & $(54.7)$ \\
\hline III & 121 & $(26.1)$ \\
\hline IV & 9 & (1.9) \\
\hline \multicolumn{3}{|l|}{ Clase funcional ingreso, $n(\%)$} \\
\hline II & 26 & $(5.6)$ \\
\hline III & 167 & $(36.1)$ \\
\hline IV & 269 & $(58.2)$ \\
\hline Hospitalización previa por $\mathrm{FC}, \mathrm{n}(\%)$ & 223 & $(47.3)$ \\
\hline \multicolumn{3}{|l|}{ Medicación previa al ingreso, n (\%) } \\
\hline Betabloqueador & 190 & $(41.1)$ \\
\hline ARA II & 163 & $(35.2)$ \\
\hline IECA & 155 & $(33.5)$ \\
\hline Antagonista de la aldosterona & 107 & $(23.1)$ \\
\hline \multicolumn{3}{|l|}{ Arritmias en EKG de ingreso, n (\%) } \\
\hline Fibrilación auricular & $94 / 461$ & (20.4) \\
\hline Flutter auricular & $12 / 461$ & $(2.6)$ \\
\hline Taquicardia supraventricular & $3 / 461$ & $(0.6)$ \\
\hline Marcapaso/cardiodesfibrilador, n (\%) & $12 / 461$ & (2.6) \\
\hline Estancia hospitalaria, días, mediana (RIQ) & 6 & $(4-10)$ \\
\hline Prolongada ( $>5$ días), $\mathrm{n}(\%)$ & 248 & $(53.9)$ \\
\hline \multicolumn{3}{|c|}{$\begin{array}{l}\text { *Definida como un valor de creatinina }>\text { de } 1.3 \mathrm{mg} / \mathrm{dL} \text {; relacion Bun/creatinina }<20 \\
\text { † hiponatremia niveles de sodio al ingreso }<135 \mathrm{mEq} / \mathrm{L}(\mathrm{mmol} / \mathrm{L} \text { ) } \\
\text { † Datos disponibles para } 422 \text { pacientes. Punto de corte de } 0.4 \mu \mathrm{g} / \mathrm{dL} \\
\text { \$ Telopéptido N-Terminal prohormona del péptidonatriurético cerebral } \\
\text { I datos disponibles para } 434 \text { pacientes } \\
\text { ** las valvulopatías se definieron de acuerdo con hallazgos ecocardiográficos aórtica } \\
\text { (insuficiencia, estenosis, doble lesión) mitral (estenosis, insuficiencia, doble lesión, } \\
\text { prótesis) tricuspídea (insuficiencia, estenosis). } \\
\text { Abreviaturas: } I E C A S \text { (inhibidores de la enzima convertidora de angiotensina). } \boldsymbol{A R A} \\
\text { (Antagonistas de los receptores de angiotensina), } \boldsymbol{F C} \text { (falla cardiaca), } \boldsymbol{r p m} \text { (respiraciones } \\
\text { por minuto), } \boldsymbol{l p m} \text { (latidos por minuto). }\end{array}$} \\
\hline
\end{tabular}


la enzima convertidora de angiotensina (IECA) y $163(35.2 \%)$ antagonistas de los receptores de angiotensina II (ARAII). La presión arterial sistólica promedio al ingreso fue de 131.6 (DE 26.4) $\mathrm{mmHg}, 375(81.1 \%)$ presentaron frecuencia cardiaca mayor de 70 lpm y 167 (36.1\%) ingresaron con clase funcional NYHA III. Entre los hallazgos paraclínicos, 47 (10.2\%) presentaron niveles de creatinina mayor de $2 \mathrm{mg} / \mathrm{dL}, 74$ (16.3\%) niveles de $\mathrm{BUN} \geq 43 \mathrm{mg} / \mathrm{dL}, 98$ (21.2\%) hiponatremia y 41 (9.7\%) troponina positiva.

En $438(97.2 \%)$ pacientes se recolectaron datos de NTproBNP al ingreso, la mediana fue de $4630 \mathrm{pcg} / \mathrm{mL}$ (Q1: <1780, Q2: 1780 a 4629, Q3: 4630 a 12068 Q4: >12068 pcg/ $\mathrm{mL})$. Se realizó una estratificación por edad de los niveles de NT-proBNP, en donde se observó que 350 (81\%) de los pacientes cumplieron criterios diagnósticos de acuerdo con la edad y nivel de NT-proBNP (Tabla 2). Se realizó ecocardiograma en 434(93.5\%), de los cuales 143 (32.9\%) tuvieron FEVI $<40 \%$.

La mediana de estancia hospitalaria fue de seis días (RIQ 4-10 días), con una estancia prolongada en 248 (53.9\%). La mortalidad intrahospitalaria y a 30 días fue de $41(8.9 \%)$ y $64(13.8 \%)$ respectivamente.

En el análisis bivariado para el desenlace mortalidad hospitalaria, las variables que tuvieron significancia estadística
Tabla 2. Valor diagnóstico de NT-proBNP para insuficiencia cardiaca de acuerdo con la edad.

\begin{tabular}{|l|c|c|}
\hline Valores de NT-proBNP & n/total & $(\%)$ \\
\hline NT-proBNP $(<50$ años $)>450 *$ & $25 / 27$ & $(92.5)$ \\
NT-proBNP $(50-75$ años $)>900 *$ & $177 / 202$ & $(87.6)$ \\
NT-proBNP $(>75$ años $)>1800 *$ & $153 / 209$ & $(73.2)$ \\
\hline *Medición en pg/mL. & \\
\hline
\end{tabular}

fueron: troponina I positiva (OR= 2.44, IC 95\% 1.22-4.91, $\mathrm{p}=0.013)$; antecedente de EPOC $(\mathrm{OR}=1.38$, IC 95\% 1.04$1.83, \mathrm{p}=0.045)$; NT-proBNP $\geq 4630 \mathrm{pg} / \mathrm{dL}(\mathrm{OR}=1.43$, IC $95 \% 1.12-1.81, \mathrm{p}=0.01)$ y $\mathrm{BUN} \geq 43 \mathrm{mg} / \mathrm{dL}(\mathrm{OR}=2.35$, IC $95 \% 1.45-3.82, \mathrm{p}=0.002)$. Para mortalidad a 30 días: estancia mayor de cinco días (OR= 1.28 [IC 95\% 1.05-1.57, $\mathrm{p}=0.02), \mathrm{BUN} \geq 43 \mathrm{mg} / \mathrm{dL}(\mathrm{OR}=2.11$, IC $95 \%$ 1.34-3.30, $\mathrm{p}=0.002)$, NT-proBNP $\geq 4630 \mathrm{pg} / \mathrm{dL}(\mathrm{OR}=1.53, \mathrm{IC} 95 \%$ $1.26-1.86, \mathrm{p}=0.004)$, troponina $(\mathrm{OR}=2.53$, IC $95 \% 1.37$ $4.69, \mathrm{p}=0.003)$ y PAS $(\mathrm{OR}=1.56$, IC $95 \% 1.07-2.27, \mathrm{p}=$ 0.02) (Tabla 3).

En el análisis multivariado para el desenlace mortalidad intrahospitalaria se observó que la variable con significancia estadística fue el BUN $\geq 43 \mathrm{mg} / \mathrm{dL}(\mathrm{OR}=3.45$, IC 95\% 1.547.74; $\mathrm{p}=0.003$ ) (Tabla 4), mientras que para mortalidad a 30

Tabla 3. Modelo bivariado para mortalidad intrahospitalaria y a 30 días, $n=462$.

\begin{tabular}{|c|c|c|c|c|}
\hline \multirow[t]{2}{*}{ Variables } & \multicolumn{2}{|c|}{ Mortalidad intrahospitalaria } & \multicolumn{2}{|c|}{ Mortalidad a 30 días } \\
\hline & OR IC 95\% & Valor de $\mathbf{P}$ & OR IC $95 \%$ & Valor de $\mathbf{P}$ \\
\hline $\begin{array}{l}\text { Edad } \\
\text { Sexo }\end{array}$ & $\begin{array}{l}1.04(0.99-1.10) \\
0.85(0.58-1.24)\end{array}$ & $\begin{array}{l}0.18 \\
0.38\end{array}$ & $\begin{array}{l}1.04(0.98-1.09) \\
0.89(0.66-1.20)\end{array}$ & $\begin{array}{l}0.07 \\
0.47\end{array}$ \\
\hline $\begin{array}{l}\text { Signos vitales } \\
\text { Frecuencia cardiaca } \geq 70 \mathrm{lpm} \\
\text { Presión arterial sistólica }<115 \mathrm{mmHg}\end{array}$ & $\begin{array}{l}1.05(0.92-1.20) \\
1.54(0.99-1.10)\end{array}$ & $\begin{array}{l}0.47 \\
0.07\end{array}$ & $\begin{array}{l}1.04(0.93-1.17) \\
1.56(1.07-2.27)\end{array}$ & $\begin{array}{l}0.47 \\
0.02\end{array}$ \\
\hline $\begin{array}{l}\text { Concentración sérica } \\
\text { Hemoglobina }<12 \mathrm{~g} / \mathrm{dL} \\
\text { Sodio }<135 \mathrm{mEq} / \mathrm{L}^{*} \\
\text { Creatinina }>2 \mathrm{mg} / \mathrm{dL} \\
\text { BUN } \geq 43 \mathrm{mg} / \mathrm{dL} \\
\text { Troponina } \mathrm{I}^{\dagger} \\
\text { NT-ProBNP } \geq 4630 \mathrm{pg} / \mathrm{dL}\end{array}$ & $\begin{array}{l}1.08(0.65-1.79) \\
1.28(0.74-2.19) \\
1.21(0.5-2.9) \\
2.35(1.45-3.82) \\
2.44(1.22-4.91) \\
1.43(1.12-1.81)\end{array}$ & $\begin{array}{c}0.75 \\
0.38 \\
0.67 \\
0.002 \\
0.013 \\
0.01\end{array}$ & $\begin{array}{l}1.03(0.67-1.58) \\
0.93(0.55-1.57) \\
1.08(0.50-2.30) \\
2.11(1.34-3.30) \\
2.53(1.37-4.69) \\
1.53(1.26-1.86)\end{array}$ & $\begin{array}{c}0.86 \\
0.8 \\
0.84 \\
0.002 \\
0.003 \\
0.004\end{array}$ \\
\hline $\begin{array}{l}\text { Comorbilidades } \\
\text { Enfermedad coronaria } \\
\text { EPOC } \\
\text { Diabetes mellitus tipo } 2 \\
\text { Fibrilación auricular } \\
\text { Hospitalización previa por FC } \\
\text { Antecedente de hipertensión arterial } \\
\text { Uso de betabloqueador previo }\end{array}$ & $\begin{array}{l}1.18(0.64-2.18) \\
1.38(1.04-1.83) \\
1.04(0.59-1.84) \\
1.35(0.79-2.33) \\
1.14(0.84-1.55) \\
0.96(0.81-1.14) \\
0.88(0.57-1.33)\end{array}$ & $\begin{array}{c}0.59 \\
0.045 \\
0.87 \\
0.28 \\
0.4 \\
0.67 \\
0.53\end{array}$ & $\begin{array}{l}1.39(0.87-2.24) \\
1.25(0.97-1.62) \\
0.92(0.56-1.51) \\
1.46(0.94-2.27) \\
1.20(0.94-1.53) \\
0.98(0.86-1.12) \\
1.02(0.75-1.40)\end{array}$ & $\begin{array}{c}0.17 \\
0.1 \\
0.75 \\
0.1 \\
0.16 \\
0.86 \\
0.86\end{array}$ \\
\hline $\begin{array}{l}\text { Variables al ingreso } \\
\text { FEVI } \leq 40 \% \\
\text { Estancia prolongada } \neq\end{array}$ & $\begin{array}{c}0.88(0.57-1.33) \\
1.14(0.88-1.48)\end{array}$ & $\begin{array}{l}0.67 \\
0.34\end{array}$ & $\begin{array}{l}1.07(0.73-1.57) \\
1.28(1.05-1.57)\end{array}$ & $\begin{array}{l}0.71 \\
0.02\end{array}$ \\
\hline $\begin{array}{l}* \text { Sodio sérico }<135 \mathrm{mEq} / \mathrm{L} \text { define hipo } \\
\dagger \text { Positiva }>0.4 \mu \mathrm{g} / \mathrm{d} \\
\text { † Hospitalización mayor de cinco días } \\
\text { Abreviaturas: FC: Falla cardiaca, FEVI }\end{array}$ & ntrículo izouierd & & & \\
\hline
\end{tabular}


días, fueron estancia hospitalaria prolongada $(\mathrm{OR}=1.98$, IC 95\% 1.04-3.75, $\mathrm{p}=0.036), \mathrm{BUN} \geq 43 \mathrm{mg} / \mathrm{dL}(\mathrm{OR}=2.23$, IC $95 \% 1.11-4.45, \mathrm{p}=0.023)$ y NT-proBNP $\geq 4630 \mathrm{pg} / \mathrm{dL}(\mathrm{OR}=$ 2.52, IC 95\% 1.25-5.08, $\mathrm{p}=0.009$ ) (Tabla 5).

\section{Discusión}

En el presente estudio se evaluaron pacientes con FC descompensada admitidos a un hospital de cuarto nivel de atención en Colombia. La descripción de esta población es semejante a la de registros internacionales (9-11), predominó el sexo femenino, el promedio de edad fue de 72.4 años, hubo alta prevalencia de enfermedades como HTA, EPOC y casi la mitad de los pacientes tuvieron antecedentes de hospitalización previa por descompensación de FC. Entre las diferencias más importantes podemos destacar el alto porcentaje de pacientes con anemia y fracción de eyección preservada.

En nuestro estudio se observó una mortalidad hospitalaria de $8.9 \%$ y a 30 días de $13.8 \%$, la cual es mayor a las reportadas en registros internacionales como el ADHERE (3.2 y $4.5 \%)$ (21) y el OPTIMIZE-HF (3.8\%)(22) y a la obtenida en un estudio realizado en nuestra institución entre 2007 y 2008 , donde fue de $4.2 \%$ (16). Sin embargo, otros registros presentan mortalidades hospitalarias más altas, en el segundo registro europeo de falla cardiaca (EHFS II) fue $6.7 \%$ (11) $\mathrm{y}$ en un registro francés de $8 \%$ (23). En Latinoamérica, en

Tabla 4. Factores relacionados a mortalidad intrahospitalaria. Análisis multivariado.

\begin{tabular}{|l|c|c|}
\hline Variables & OR $($ IC 95\%) & valor $\boldsymbol{p}$ \\
\hline Edad & $1.63(0.82-3.23)$ & 0.155 \\
Sexo & $0.49(0.22-1.06)$ & 0.073 \\
Antecedente de EPOC & $1.53(0.71-3.28)$ & 0.268 \\
PAS ingreso* & $1.66(0.75-3.65)$ & 0.203 \\
BUN $\dagger$ & $3.45(1.54-7.74)$ & 0.003 \\
Troponina I positiva $¥$ & $1.25(0.42-3.73)$ & 0.684 \\
NT-ProBNP § & $2.13(0.90-5.03)$ & 0.082 \\
\hline * Tensión arterial sistólica menor de $115 \mathrm{mmHg}$ & \\
$\dagger$ Definido como BUN $\geq$ de $43 \mathrm{mg} / \mathrm{dL}$ \\
$\begin{array}{l}\text { Punto de corte de } 0.4 \mu \mathrm{dL} \\
\S \text { Niveles } \geq 4630 \mathrm{pg} / \mathrm{mL}\end{array}$
\end{tabular}

Tabla 5. Factores relacionados a mortalidad a 30 días. Análisis multivariado.

\begin{tabular}{|l|c|c|}
\hline Variables & OR $($ IC 95\%) & valor $\boldsymbol{p}$ \\
\hline BUN* & $2.23(1.11-4.45)$ & 0.023 \\
Estancia prolongada $\dagger$ & $1.98(1.04-3.75)$ & 0.036 \\
NT-ProBNP $\ddagger$ & $2.52(1.25-5.08)$ & 0.009 \\
Troponina I positiva & $1.52(0.64-3.62)$ & 0.334 \\
PAS ingreso $\$$ & $1.36(0.69-2.67)$ & 0.365 \\
\hline *Definido como BUN $\geq$ de 43mg/dL & \\
†Estancia $\geq 5$ días & \\
†Niveles $\geq 4630 \mathrm{pg} / \mathrm{ml}$ & \\
§PAS $>115 \mathrm{mmHg}$ & \\
\hline
\end{tabular}

el registro chileno ICARO fue del 5.6\% (12), mientras que en el registro argentino de falla cardiaca $8 \%$ (13).

Uno de los factores que puede influir en esta mortalidad es la estancia hospitalaria, ya que los registros que muestran mortalidades bajas presentan estancias hospitalarias cortas (4.3 días en el registro ADHERE) (21). Cuando se evalúa la mortalidad a 30 días, la mortalidad está entre 8 y $11 \%$, las cuales son más próximas a la encontrada en este estudio (24). Adicionalmente algunos registros han sido diseñados con el objetivo de mejorar las medidas de desempeño del tratamiento hospitalario. En éstos las mortalidades son bajas y es probable que refleje la aplicación más adecuadas de tratamientos basado en la evidencia $(22,25)$. En el presente estudio no fue posible valorar la adherencia a las medidas de desempeño recomendadas por las diferentes guías de manejo, por lo que no se puede descartar que la mortalidad esté relacionada con un menor cumplimiento de estas recomendaciones. Sin embargo, un análisis realizado en 47 pacientes de esta cohorte mostró un cumplimiento de guías al egreso entre 52.6 y $68.4 \%$ en la formulación de IECAS o ARAII, BB y espironolactona. El principal error de formulación encontrado fue el uso de metoprolol tartrato (26).

Tampoco se pueden descartar factores sociales y/o biológicos que condicione a esta población de estudio a una mayor mortalidad. Finalmente la mortalidad encontrada fue mayor con respecto a una medición en nuestra institución realizada en 2007-2008 (16). Entre las diferencias más importantes, en la presente investigación el promedio de edad fue mayor, así como la severidad de la descompensación al ingreso ya que un alto porcentaje ingresaron con clase funcional IV y muy pocos con clase II. Esto puede ser una explicación en parte de la mayor estancia hospitalaria vista en el presente estudio, lo cual también influye en la mortalidad. Algunas otras diferencias no pueden ser comparadas (FE y NTproBNP). De todas formas no hay otras causas evidentes que expliquen estas diferencias.

En cuanto a los predictores de mortalidad, estudios previos han identificado variables relacionadas con la mortalidad en pacientes hospitalizados por FC (27-29). La presión arterial sistólica, función renal alterada y enfermedades crónicas como la EPOC, constituyen predictores importantes de mortalidad y se asocian a una mayor tasa de complicaciones y pobres resultados $(27,30,31)$. En nuestro caso, el BUN $\geq$ $43 \mathrm{mg} / \mathrm{dL}$ tuvo una asociación con mortalidad hospitalaria y a 30 días, mientras que el pro-BNP $>4630 \mathrm{pg} / \mathrm{dL}$ y la estancia prolongada se asociaron con mortalidad a 30 días. Otras variables no mostraron significancia estadística.

La troponina I positiva fue factor de riesgo para mortalidad en el análisis bivariado, pero no alcanzó significancia estadística en el análisis multivariado de mortalidad a 30 días. Esto es consistente con otros estudios en donde la troponina no alcanza significancia estadística en el análisis multivariado $(32,33)$. En este caso, diferencias en las poblaciones de estudio, la medición (tipo, generación de ensayo, punto de corte, centro hospitalario de estudio) y la 
mezcla de datos de troponina I y $\mathrm{T}$ afectan los resultados. Además en falla cardiaca aguda la elevación de la troponina es multifactorial y en muchas ocasiones es por causas reversibles (sepsis, arritmias, anemia), por lo que aún no está bien definida la utilidad de la medición única o seriada de la troponina en estos pacientes (34).

Recientemente los péptidos natriuréticos (BNP y NTproBNP) se han relacionado con el pronóstico a corto y largo plazo en pacientes con FC $(35,36)$. Se ha establecido que un valor $\geq 5.000 \mathrm{mg} / \mathrm{mL}$ se asocia con un incremento de la mortalidad intrahospitalaria y mayor estancia hospitalaria en falla cardiaca aguda (37). En nuestro caso, se realizó un análisis del NT-proBNP valores mayores que la mediana tuvieron asociación estadísticamente significativa para mortalidad a 30 días. Esto es consistente con otros resultados en donde los cuartiles de péptidos natriuréticos son un factor de riesgo de mortalidad (38).

En muchos estudios se ha encontrado que la alteración en la función renal se asocia con mayor riesgo de mortalidad, estancia prolongada y tasas de readmisión en pacientes con FC. Se ha establecido que el BUN y el cociente BUN/creatinina son mejores predictores de mortalidad intrahospitalaria en el paciente con falla cardiaca aguda comparado con la creatinina y la disminución de la depuración de creatinina, los cuales son mejores predictores de mortalidad en el paciente ambulatorio estable $(39,40)$. En nuestro caso, un $\mathrm{BUN} \geq 43 \mathrm{mg} / \mathrm{dL}$ fue el principal factor de riesgo asociado a mortalidad hospitalaria y a 30 días, mientras que la creatinina $>2 \mathrm{mg} / \mathrm{dL}$, no tuvo asociación con estos resultados.

Esta es una investigación con un gran número de pacientes, que permite analizar en nuestra población distintas variables identificadas en otros estudios asociadas con mortalidad hospitalaria y a 30 días en pacientes con FC descompensada. El diseño del estudio y el tamaño de la muestra permiten obtener datos confiables y representativos de la población que asiste a nuestro hospital. Gracias a estas observaciones y resultados tenemos la base para definir estrategias que contribuyan a mejorar la atención de los pacientes, con potencial impacto favorable en la mortalidad y los costos, con lo que se puede generar el deseado "impacto social" que debe tener una investigación.

En cuanto a las limitaciones, el estudio es unicéntrico, por lo que no necesariamente demuestra la realidad global de la FC descompensada en Colombia. No se hizo una distinción entre los pacientes hospitalizados en salas generales de hospitalización y aquellos que requirieron manejo en UCI, en los cuales hay otros factores que influyen en los resultados.

\section{Conclusión}

La población de pacientes con falla cardiaca descompensada evaluada en este estudio es semejante a la descrita en otros estudios poblacionales, la mortalidad intrahospitalaria fue elevada, aunque cercana a la de otros registros latinoamericanos. En los análisis multivariados, se encontró que el nitrógeno ureico $\geq 43 \mathrm{mg} / \mathrm{dL}$ es el único factor de riesgo asociado a mortalidad intrahospitalaria, mientras que la mortalidad a 30 días se relacionó además del nitrógeno ureico aumentado con el NT-proBNP elevado y la estancia hospitalaria superior a cinco días.

\section{Agradecimientos}

A los residentes de medicina interna. Ruth Castiblanco Montañez, Coordinadora del proyecto; César Piñeros Perilla, Estadístico División de Investigaciones.

\section{Conflicto de interés}

Ninguno.

\section{Referencias}

1. Go AS, Mozaffarian D, Roger VL, Benjamin EJ, Berry JD, Blaha MJ, et al. Heart disease and stroke statistics--2014 update: a report from the American Heart Association. Circulation. 2014; 129(3): e28-e292.

2. McMurray JJ, Adamopoulos S, Anker SD, Auricchio A, Böhm M, Dickstein $\mathbf{K}$, et al. ESC Guidelines for the diagnosis and treatment of acute and chronic heart failure 2012: The Task Force for the Diagnosis and Treatment of Acute and Chronic Heart Failure 2012 of the European Society of Cardiology. Developed in collaboration with the Heart Failure Association (HFA) of the ESC. Eur Heart J. 2012; 33(14): 1787-847.

3. Yancy CW, Jessup M, Bozkurt B, Butler J, Casey DE, Drazner MH, et al. 2013 ACCF/AHA guideline for the management of heart failure: a report of the American College of Cardiology Foundation/American Heart Association Task Force on Practice Guidelines. J Am Coll Cardiol. 2013; 62(16): 147-239.

4. Cubillos-Garzón LA, Casas JP, Morillo CA, Bautista LE. Congestive heart failure in Latin America: the next epidemic. Am Heart J. 2004; 147(3): 412-7.

5. Hernández-Leiva E. [Epidemiology of acute coronary syndrome and heart failure in Latin America]. Rev Esp Cardiol. 2011; 64 Suppl 2: 34-43.

6. Schargrodsky H,Hernández-Hernández R, Champagne BM,Silva H, Vinueza R, Silva Ayçaguer LC, et al. CARMELA: assessment of cardiovascular risk in seven Latin American cities. Am J Med. 2008; 121(1): 58-65.

7. Bocchi E, Arias A, Verdejo H, Diez M, Gómez E, Castro P. The Reality of Heart Failure in Latin America. JACC. 2013; 62(11): 949-58.

8. Stewart S, MacIntyre K, Hole DJ, Capewell S, McMurray JJ. More 'malignant' than cancer? Five-year survival following a first admission for heart failure. Eur J Heart Fail. 2001; 3(3): 315-22.

9. Adams KF, Fonarow GC, Emerman CL, LeJemtel TH, Costanzo MR, Abraham WT, et al. Characteristics and outcomes of patients hospitalized for heart failure in the United States: rationale, design, and preliminary observations from the first 100,000 cases in the Acute Decompensated Heart Failure National Registry (ADHERE). Am Heart J. 2005; 149(2): 209-16.

10. Fonarow GC, Stough WG, Abraham WT, Albert NM, Gheorghiade M, Greenberg BH, et al. Characteristics, treatments, and outcomes of patients with preserved systolic function hospitalized for heart failure: a report from the OPTIMIZE-HF Registry. J Am Coll Cardiol. 2007; 50(8): 768-77.

11. Nieminen MS, Brutsaert D, Dickstein K, Drexler H, Follath F, Harjola VP, et al. EuroHeart Failure Survey II (EHFS II): a survey on hospitalized acute heart failure patients: description of population. Eur Heart J. 2006; 27(22): 2725-36.

12. Castro G P, Verdejo P H, Vukasovic R JL, Garcés E, González I, ICARO G. Predictores de mortalidad intrahospitalaria y hospitalizacion prolongada en hospitales Chilenos. Rev Med Chil. 2006; 134(9): 1083-91.

13. Fairman E, Thierer J, Rodríguez I, Blanco P, Guetta J, Fernández S, et al. Registro Nacional de Internación por Insuficiencia Cardíaca 2007. Rev Arg Cardiol. 2009; 77(1): 33 .

14. Ospina A, Gammarra G. Características clínicas y epidemiológicas de la insuficiencia cardiaca en el Hospital Universitario Ramón Gonzalez Valencia de Bucaramanga, Colombia. Revista Salud UIS. 2004; 36(3): 125-31.

15. Senior J, Saldarriaga C, Rendon J. Descripción clínico-epidemiológica de los pacientes con falla cardiaca aguda que consultan al servicio de urgencias. Acto medica Colombiana. 2011; 36(3): 125-9.

16. Lancheros A, Valencia Y, Chaves W. Insuficiencia Cardíaca Aguda: Factores asociados con mortalidad Hospital de San José, julio 2007 - agosto 2008 Bogotá D.C. Colombia. Repert.med.cir.2009; 18(3): 166-174.

17. Castaño J, Giraldo J, Herrera H, Jaramillo J, Noreña J, Restrepo M. Caracterización de pacientes con insuficiencia cardiaca en el primer nivel de atención de la ciudad de Manizalez (colombia) 2005-2008. Archivos de Medicina. 2010; 10(2): $127-38$

18. McKee PA, Castelli WP, McNamara PM, Kannel WB. The natural history of 
congestive heart failure: the Framingham study. N Engl J Med. 1971; 285(26): 1441-6.

19. Hsieh FY. Sample size tables for logistic regression. Stat Med. 1989; 8(7): 795802.

20. Ortega Calvo M, Cayuela Domínguez A. Regresión logística no condicionada y tamaño de muestra: una revisión bibliográfica. Rev Esp Salud Publica 2002; 76: $85-93$.

21. Fonarow GC, Heywood JT, Heidenreich PA, Lopatin M, Yancy CW. Temporal trends in clinical characteristics, treatments, and outcomes for heart failure hospitalizations, 2002 to 2004: findings from Acute Decompensated Heart Failure National Registry (ADHERE). Am Heart J. 2007; 153(6): 1021-8.

22. Fonarow GC, Abraham WT, Albert NM, Gattis Stough W, Gheorghiade $\mathbf{M}$, Greenberg BH, et al. Influence of a performance-improvement initiative on quality of care for patients hospitalized with heart failure: results of the Organized Program to Initiate Lifesaving Treatment in Hospitalized Patients With Heart Failure (OPTIMIZE-HF). Arch Intern Med. 2007; 167(14): 1493-502.

23. Tribouilloy C, Rusinaru D, Leborgne L, Mahjoub H, Szymanski C, Houpe D, et al. In-hospital mortality and prognostic factors in patients admitted for new-onset heart failure with preserved or reduced ejection fraction: a prospective observational study. Arch Cardiovasc Dis. 2008; 101(4): 226-34.

24. Bueno H, Ross JS, Wang Y, Chen J, Vidán MT, Normand SL, et al. Trends in length of stay and short-term outcomes among Medicare patients hospitalized for heart failure, 1993-2006. JAMA. 2010; 303(21): 2141-7.

25. Heidenreich PA, Lewis WR, LaBresh KA, Schwamm LH, Fonarow GC. Hospital performance recognition with the Get With The Guidelines Program and mortality for acute myocardial infarction and heart failure. Am Heart J. 2009; 158(4): 546-53.

26. Chaves WG, Diaztagle JJ, Vargas V, Mejía MG, Sprockel JJ, Hernández JI. Cumplimiento de guías en pacientes hospitalizados con falla cardiaca ¿Cómo estamos?. Act Med Colom 2014; 39:1 (en prensa).

27. O'Connor CM, Abraham WT, Albert NM, Clare R, Gattis Stough W, Gheorghiade $\mathbf{M}$, et al. Predictors of mortality after discharge in patients hospitalized with heart failure: an analysis from the Organized Program to Initiate Lifesaving Treatment in Hospitalized Patients with Heart Failure (OPTIMIZE-HF). Am Heart J. 2008; 156(4): 662-73.

28. Lee DS, Stitt A, Austin PC, Stukel TA, Schull MJ, Chong A, et al. Prediction of heart failure mortality in emergent care: a cohort study. Ann Intern Med. 2012; 156(11): 767-75, W-261, W-2.

29. Pocock SJ, Wang D, Pfeffer MA, Yusuf S, McMurray JJ, Swedberg KB, et al. Predictors of mortality and morbidity in patients with chronic heart failure.
Eur Heart J. 2006; 27(1): 65-75.

30. Lee DS, Austin PC, Rouleau JL, Liu PP, Naimark D, Tu JV. Predicting mortality among patients hospitalized for heart failure: derivation and validation of a clinical model. JAMA. 2003; 290(19): 2581-7.

31. Harjai KJ, Thompson HW, Turgut T, Shah M. Simple clinical variables are markers of the propensity for readmission in patients hospitalized with heart failure. Am J Cardiol. 2001; 87(2): 234-7, A9.

32. Ilva T, Lassus J, Siirilä-Waris K, Melin J, Peuhkurinen K, Pulkki K, et al. Clinical significance of cardiac troponins $\mathrm{I}$ and $\mathrm{T}$ in acute heart failure. Eur $J$ Heart Fail. 2008; 10(8): 772-9.

33. Sakhuja R, Green S, Oestreicher EM, Sluss PM, Lee-Lewandrowski E, Lewandrowski KB, et al. Amino-terminal pro-brain natriuretic peptide, brain natriuretic peptide, and troponin $\mathrm{T}$ for prediction of mortality in acute heart failure. Clin Chem. 2007; 53(3): 412-20.

34. Januzzi JL, Filippatos G, Nieminen M, Gheorghiade M. Troponin elevation in patients with heart failure: on behalf of the third Universal Definition of Myocardial Infarction Global Task Force: Heart Failure Section. Eur Heart J. 2012; 33(18): 2265-71.

35. van Kimmenade RR, Pinto YM, Bayes-Genis A, Lainchbury JG, Richards AM, Januzzi JL. Usefulness of intermediate amino-terminal pro-brain natriuretic peptide concentrations for diagnosis and prognosis of acute heart failure. Am J Cardiol. 2006; 98(3): 386-90.

36. Anand IS, Fisher LD, Chiang YT, Latini R, Masson S, Maggioni AP, et al. Changes in brain natriuretic peptide and norepinephrine over time and mortality and morbidity in the Valsartan Heart Failure Trial (Val-HeFT). Circulation. 2003; 107(9): 1278-83.

37. Murtagh G, Canniffe C, Mahgoub M, Blake L, McCarroll N, Crowley V, et al. Introduction of an NT-proBNP assay to an acute admission unit--a 2-year audit. Eur J Intern Med. 2009; 20(1): 58-62.

38. Fonarow GC, Peacock WF, Phillips CO, Givertz MM, Lopatin M, Investigators ASACa.Admission B-type natriuretic peptide levels and in-hospital mortality in acute decompensated heart failure. J Am Coll Cardiol. 2007; 49(19): 1943-50.

39. Klein L, Massie BM, Leimberger JD, O'Connor CM, Piña IL, Adams KF, et al. Admission or changes in renal function during hospitalization for worsening heart failure predict postdischarge survival: results from the Outcomes of a Prospective Trial of Intravenous Milrinone for Exacerbations of Chronic Heart Failure (OPTIME-CHF). Circ Heart Fail 2008; 1(1): 25-33.

40. Logeart D, Tabet JY, Hittinger L, Thabut G, Jourdain P, Maison P, et al. Transient worsening of renal function during hospitalization for acute heart failure alters outcome. Int J Cardiol. 2008; 127(2): 228-32. 